\title{
Tunable Fermi acceleration in a nondissipative driven magnetic billiard
}

\author{
B. Castaldi, ${ }^{1}$ R. Egydio de Carvalho, ${ }^{1}$ C. Vieira Abud, ${ }^{2}$ and A. P. Mijolaro ${ }^{3}$ \\ ${ }^{1}$ Universidade Estadual Paulista-UNESP, Rio Claro-SP, Brazil \\ ${ }^{2}$ Universidade de São Paulo-USP, São Paulo-SP, Brazil \\ ${ }^{3}$ Centro Estadual de Educação Tecnológica-CEETEPS, Rio Claro-SP, Brazil
}

(Received 14 October 2013; published 21 January 2014)

\begin{abstract}
We study the effect of a constant magnetic field on the dynamics of a system that may present Fermi acceleration (FA). The model in consideration is the nondissipative annular billiard with breathing boundaries. There is a field threshold, from which the mechanism of FA can be deactivated. The presence of the magnetic field curves the particle trajectories and for some combinations of the parameters FA is totally, and nontrivially, suppressed without considering any kind of dissipation.
\end{abstract}

DOI: 10.1103/PhysRevE.89.012916

PACS number(s): 05.45.Pq, 05.45.Gg, 45.20.Jj

\section{INTRODUCTION}

In recent years, several studies have been developed in order to show dynamical properties which could contribute for inducing or suppressing Fermi acceleration (FA), which can be named as indefinite energy growth [1-18]. The Loskutov-Ryabov-Akinshin conjecture says that a chaotic regime observed in static billiards is a sufficient condition to find FA when a periodic time-dependent perturbation is added on the billiard boundaries [1]. This conjecture has been verified in many works and also expanded when there is a separatrix in the static case instead of a chaotic dynamics [7]. On the other hand, other works have presented studies on how robust FA is under the action of dissipation at the collisions with the boundaries or through some kind of viscosity $[5,8]$. Contrarily to these approaches, in this present work we report the suppression of FA without introducing any kind of dissipation, but by applying a constant magnetic field.

In general, billiards consist of appropriated models to study physical phenomena or properties of more complex systems. In the particular focus of this work, the mechanism of FA has been noticed in the stadiumlike billiard $[2,9,11,12]$, in the annular billiard $[3,4,10]$, in the one-dimensional stochastic Fermi-Ulam billiard $[5,6]$, in the elliptical billiard $[7,8]$, in the Sinai's billiard $[9,11]$, and in the Lorentz gas-like billiard [16]. Recent results on FA associated with the interaction of charge particles with magnetic islands or mirrors have also been reported [14,15,17,18].

The pulsating annular billiard (PAB) is the model we are going to consider to develop our study. It is a two-dimensional system limited by two breathing circular boundaries of radius $R(t)$ and $r(t)(R>r)[3,4]$. The distance between both centers is the eccentricity $\delta$, which plays the role of perturbation parameter. A particle can move freely of potential between the two collision zones, defined by $\left[R_{0}+e_{R}, R_{0}-e_{R}\right]$ and $\left[r_{0}+e_{r}, r_{0}-e_{r}\right]$ in which $R_{0}$ and $r_{0}$ are the static radii of the circles and $e_{R}$ and $e_{r}$ are the amplitude of oscillations of the boundaries. The dependence on time makes the energy no longer constant. If in addition, in the PAB, the circles are concentric, the angular momentum of the particle is a constant of motion and the phase space presents some regular structures, but if the circles are eccentric it is possible to observe the mechanism of FA. It is worth pointing out that the applied realization of the static annular billiard is reported theoretically
[13] and experimentally [19]. The paper is organized as follows: In Sec. II we present the main concepts of the magnetic pulsating annular billiard, in Sec. III we present and discuss the results as well as the conclusions, and closing the work the Appendix shows the steps between Eqs. (1) and (2).

\section{THE MAGNETIC BILLIARD}

This study is a natural sequence of previous papers [3,4,20-23], but in the present work, we now consider a charged particle, which beyond being subject to the dynamic conditions of the PAB, is also subjected to the action of a constant magnetic field perpendicularly orientated in relation to the plane of the billiard. This system will be called as magnetic pulsating annular billiard (MPAB). A schematic view of the billiard is shown in Fig. 1.

Contrarily to the usual, we do not consider a discrete map to describe the dynamics, but a continuous flux governed by the following Hamiltonian function:

$$
\mathcal{H}(\overrightarrow{\mathbf{r}}, \overrightarrow{\mathbf{p}})=\frac{1}{2 m}\left[\overrightarrow{\mathbf{p}}-\frac{q}{c} \overrightarrow{\mathbf{A}}(\overrightarrow{\mathbf{r}})\right]^{2},
$$

where $\overrightarrow{\mathbf{A}}$ is the magnetic potential vector, $m$ is the particle mass, $\overrightarrow{\mathbf{p}}$ is the total momentum of the particle, $q$ is the electric charge, and $c$ is the speed of light. Using cylindrical polar coordinates $(\rho, \theta), \overrightarrow{\mathbf{p}}=p_{\theta} \hat{\theta}+p_{\rho} \hat{\rho}$, and choosing an antisymmetric gauge, $A_{\theta}=\frac{1}{2} B \rho$, we get the constant magnetic field $\overrightarrow{\mathbf{B}}=B \hat{\mathbf{z}}$. After some algebraic manipulation, we write the Hamiltonian for the system (see Appendix),

$$
\mathcal{H}=\frac{1}{2 m}\left[p_{\rho}^{2}+\frac{1}{\rho^{2}}\left(p_{\theta}-\frac{q B \rho^{2}}{2 c}\right)^{2}\right] .
$$

This Hamiltonian represents the total energy $E$ of the particle, which is stroboscopically constant and between two successive collisions with the boundaries the total velocity of the particle is $v=\sqrt{\frac{2 E}{m}}$, which is also constant. On the other hand, when a collision occurs with any boundary there will be corrections in the radial velocity and in the particle energy. The corrections in the radial particle velocity are

$$
\begin{aligned}
& v_{R}=-v_{R}^{0}-2 e_{R} \sin \left(\phi_{e}\right), \\
& v_{r}=-v_{r}^{0}-2 \omega e_{r} \sin \left(\phi_{i}\right),
\end{aligned}
$$




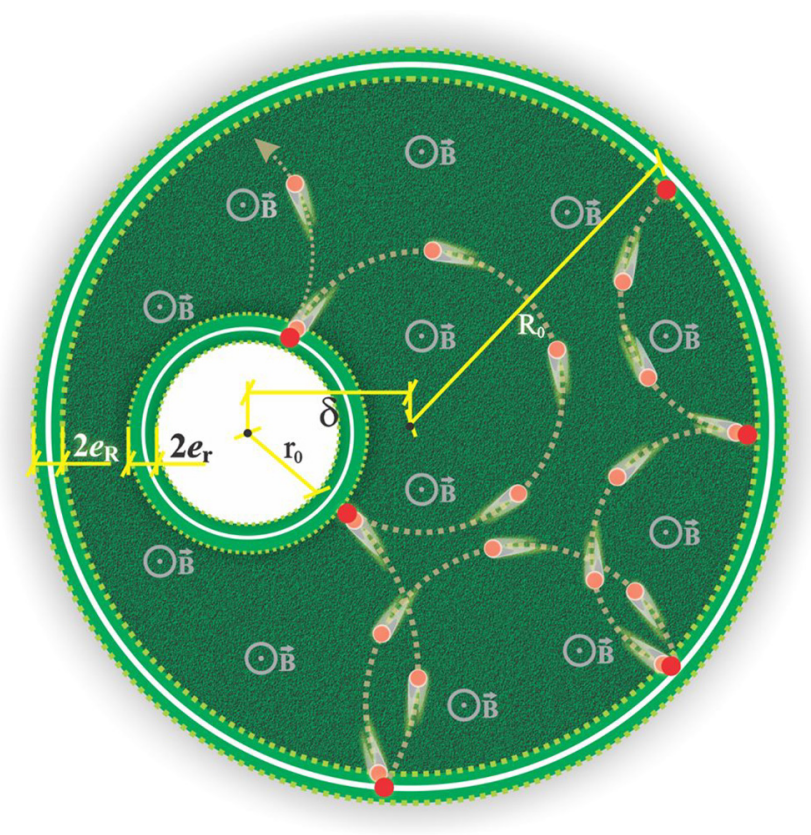

FIG. 1. (Color online) Illustration of the magnetic pulsating annular billiard with circular trajectories. The regions $\left[R_{0}+e_{R}\right.$, $\left.R_{0}-e_{R}\right]$ and $\left[r_{0}+e_{r}, r_{0}-e_{r}\right]$ are called collision zones, where $e_{R}$ and $e_{r}$ are the half amplitudes of oscillation and $R_{0}$ and $r_{0}$ are the static radii of the external and internal boundaries, respectively. The boundaries' oscillations can furnish energy to the particle during a collision, increasing its radial velocity.

where $v_{R}^{0}$ and $v_{R}$ are the radial velocities of the particle with respect to the center of the external circle immediately before and after the collision, respectively, and $v_{r}^{0}$ and $v_{r}$ the radial velocities with respect to the center of the internal circle immediately before and after the collision, respectively. $\phi_{e}$ and $\phi_{i}$ are the phase of oscillation of external and internal boundaries, respectively, at the moment of the collision and $\omega$ is the ratio of the frequencies of oscillations of both boundaries.

For the computational purposes, all calculations have been done through numeric integration of the Hamilton equations obtained from the Hamiltonian (2) using the sixth order RungeKutta integrator. A rather delicate detail here is how to find the time in the instant when a collision occurs. When the magnetic field is turned on, the particle develops a circular trajectory, whose radius of curvature is $\xi=\frac{m v}{q B}$, and for each iteration, we check if there occurs an intersection of this trajectory with the time-dependent circular boundaries, within a preset precision. At the instant that the intersection is with the outer circle we store a collision and restart the time counter. We point out that we have considered the complete model, this is, by the time the particle goes into a collision zone it can suffer successive collisions with the boundary, and we used $R_{0}=1$ for all numeric calculations.

For the static concentric geometry, $\delta=0$, with or without magnetic field, the system is globally integrable because the total energy and the angular momentum, $p_{\theta}$, are constants of motion. In the concentric pulsating case the total energy is not conserved anymore, but $p_{\theta}$ is still a constant of motion since $\theta$ is a cyclic variable. The corresponding Poincaré's section in

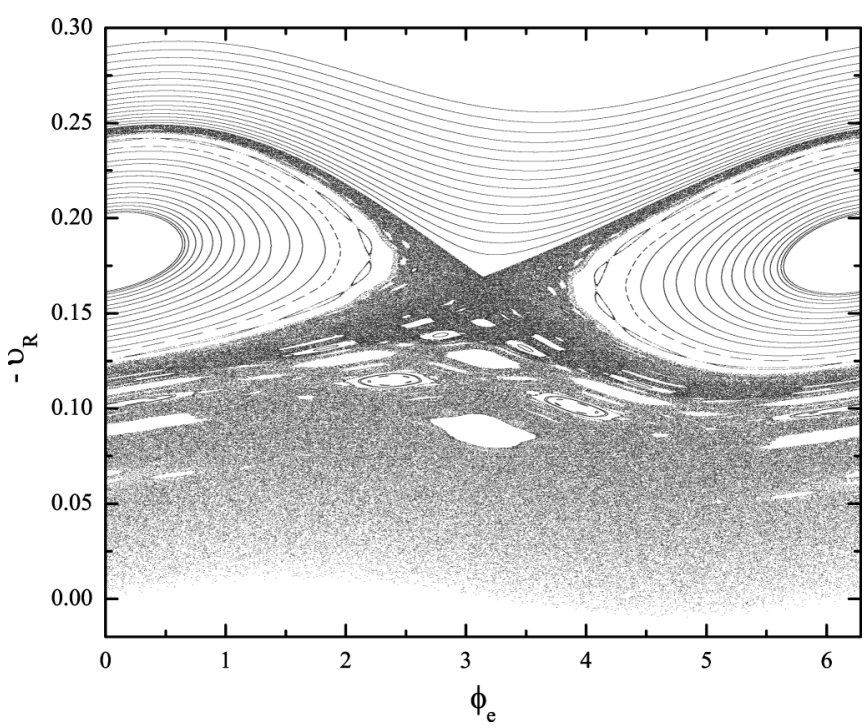

FIG. 2. Poincaré's section for $\delta=0, r_{0}=0.45, e_{R}=e_{r}=0.01$, $\omega=1.0, \phi_{e}-\phi_{i}=0$, and $B=0$. From the first invariant spanning curve and above, the movement is regular; thus only a limited growth of energy is possible for initial conditions starting inside the chaotic region.

the coordinates $\left(-v_{R}, \phi_{e}\right)$, for $B=0$, shows chaos and regular structures (Fig. 2). Thus, the growth of energy is limited to the extension of the chaotic sea.

In Fig. 3, we present Poincaré's sections for the concentric, $\delta=0$, and breathing magnetic billiard for (a) $B=0.10$; (b) $B=0.20$; (c) $B=0.40$; (d) $B=0.50$. We observe that as stronger the field as smaller the chaotic region, turning the dynamics more regular.

\section{RESULTS, DISCUSSION, AND CONCLUSIONS}

In the eccentric and pulsating scenario, as the total particle energy $E$ as $p_{\theta}$ are no longer preserved and the mechanism of indefinite energy growth (FA) has been already reported in previous works $[3,4]$. Now we are going to study the effects of the magnetic field on the phenomenon of FA. We took some known values of the parameters of the eccentric geometry without magnetic field, for which FA has been previously observed-this means $\delta=0.30, r_{0}=0.45, e_{R}=e_{r}=0.01$, $\omega=1.0$, and $\phi_{e}-\phi_{i}=0$ - and we apply a magnetic field for different magnitudes. Figure 4 shows the evolution of the particle velocity, for an ensemble of 500 initial conditions, for some values of magnetic field until $10^{5}$ iterations $(n)$.

We can observe that for weak field, $B=0.20$, the growth of the mean velocity is very similar to the case without magnetic field, while for intermediate fields, $B=0.31$ and $B=0.32$, only after $\sim 10^{2}$ iterations we can also identify some similarity in the slope of the curves with the null field case. Nevertheless, for strong fields, $B=0.45$ and $B=0.60$, the behavior of the curves is very different and FA is completely suppressed. For a deeper study, Fig. 5 shows the mean particle velocity for the same ensemble of initial conditions (IC) and the same set of parameters of Fig. 4. We plot only the last iteration, i.e., the $10^{5}$-th iteration, but now for 65 different values of magnetic field. 


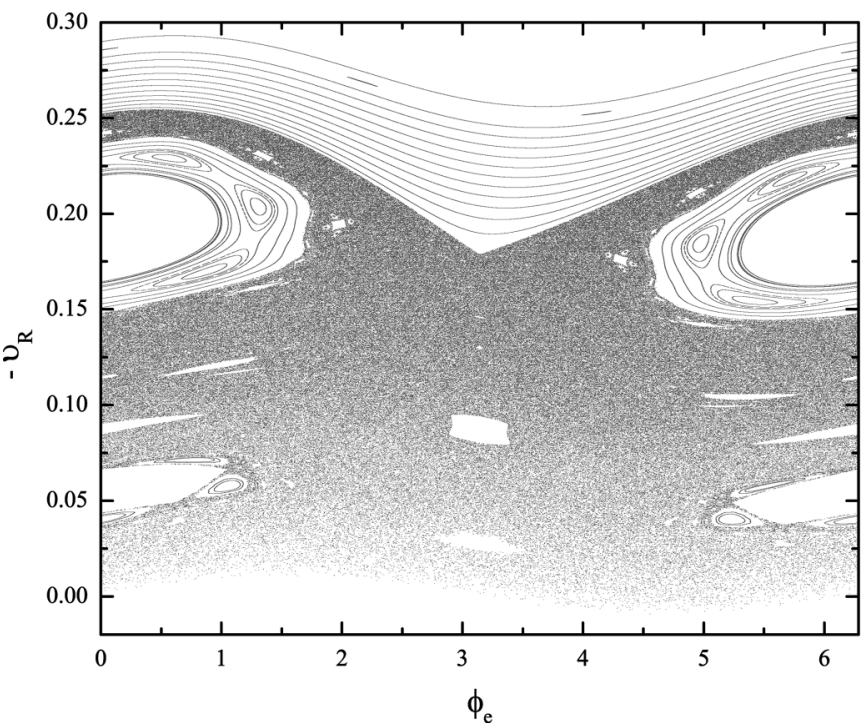

(a)

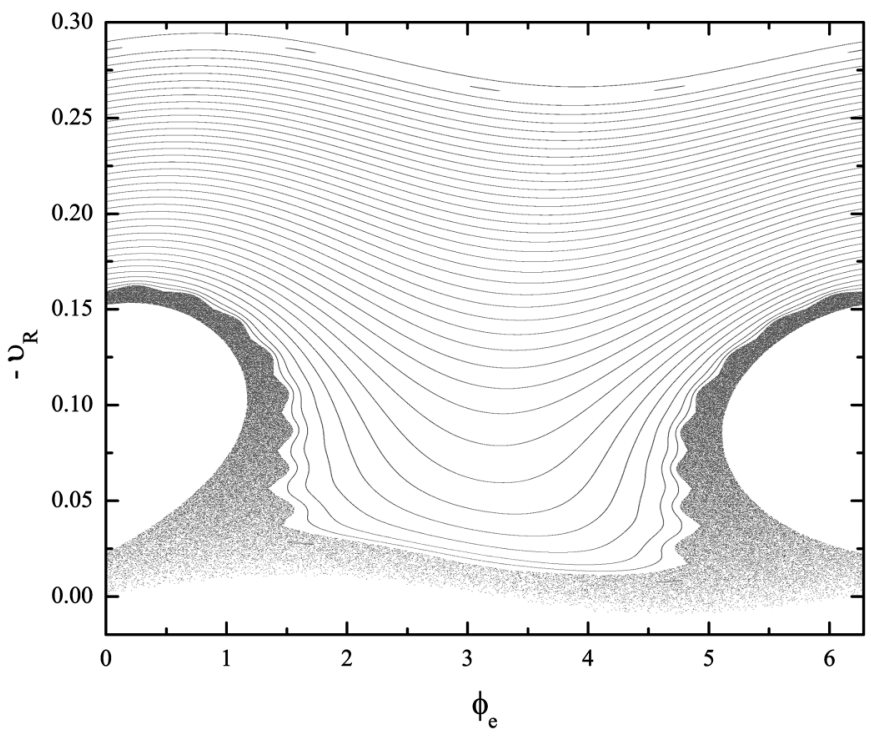

(c)

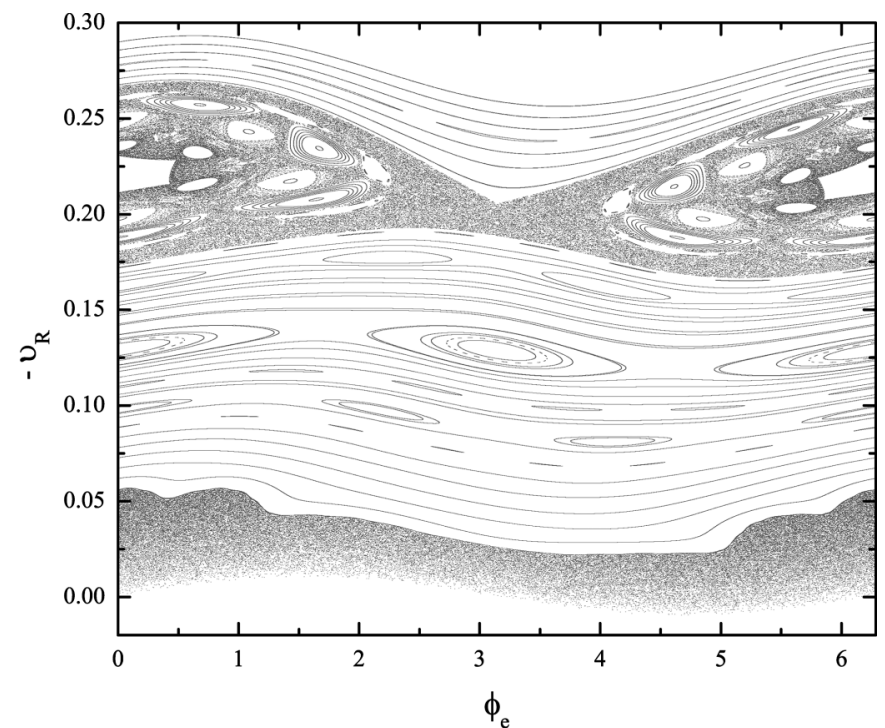

(b)

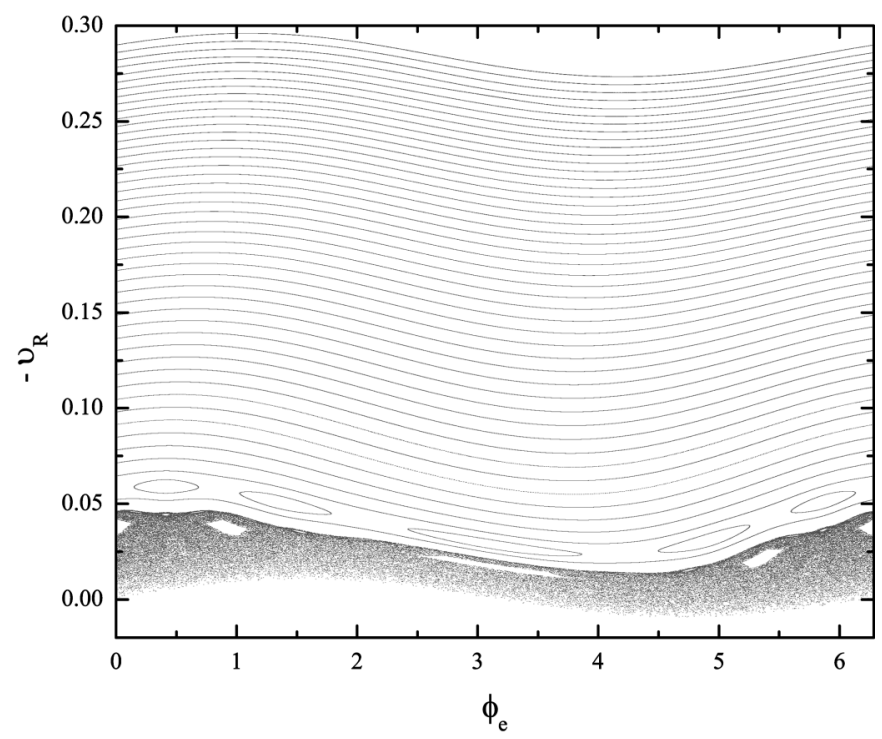

(d)

FIG. 3. Poincaré sections for $\delta=0, r_{0}=0.45, e_{R}=e_{r}=0.01, \omega=1.0, \phi_{e}-\phi_{i}=0$, and (a) $B=0.10$, (b) $B=0.20$, (c) $B=0.40$, (d) $B=0.50$. The regularity increases with the increasing of $B$.

The red line is a nonlinear fitting given by the classic fourparametric sigmoid Boltzmann function, commonly used for sigmoidal datasets and phase transition models [24], obtained with an adjusted rate of $99.4 \%$, and here it can be described as

$$
\left\langle v_{M}\right\rangle=f(B)=\frac{\left\langle v_{M}\right\rangle_{i}-\left\langle v_{M}\right\rangle_{f}}{1+e^{\frac{B-B_{C}}{\Delta B}}}+\left\langle v_{M}\right\rangle_{f},
$$

where $\left\langle v_{M}\right\rangle_{f}=0.03 \pm 0.01$ is the value of the mean velocity for $B=0.6$, while $\left\langle v_{M}\right\rangle_{i}=1.34 \pm 0.01$ is for $B=0$. $B_{C}=0.3139 \pm 0.0006$ is the central point of the transition decay, $\Delta B=0.0064 \pm 0.0005$ is the step of values of $B$, and $M$ symbolizes the final iteration, $n=10^{5}$. It is important to notice that this estimate is valid for the $10^{5}$-th iteration and it may be changed for other amounts of iterations. In spite of that, it illustrates some generic aspects of the influence of the action of the magnetic field. For small and intermediate values of $B$ the particle can reach velocities higher than the initial velocity, $v_{0}=0.02$, and FA can be observed. Around $B \sim 0.30$ and $B \sim 0.33$ there are two transitions; the first one suggests approximately the beginning of the suppression of FA and the second one corresponds to a new plateau of saturation indicating the confinement of the particle in regions of the phase space with low velocities. Even though we are considering the eccentric case, these results are in total agreement with the plots of Fig. 3, for the concentric geometry, concerning the effect of the magnetic field.

Figures 4 and 5 show only up to $10^{5}$ collisions with the external boundary because the integration of the Hamilton equations spends too much computational time. However, in order to not have any doubt about the results, we present in Fig. 6 the calculation of four strong values of the magnetic field, $B=0.45, B=0.50, B=0.55$, and $B=0.60$, up $10^{8}$ 


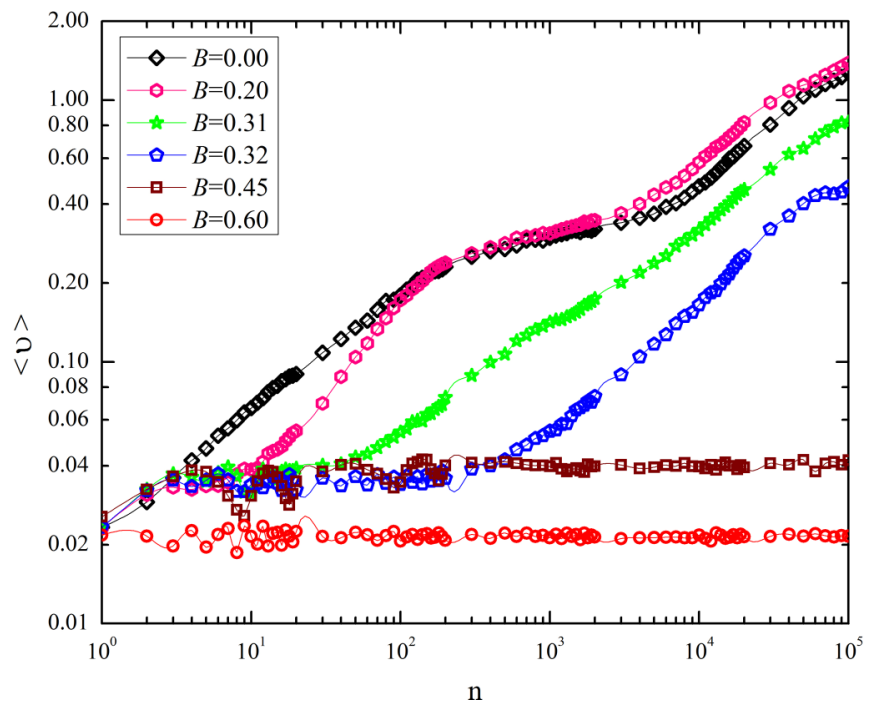

FIG. 4. (Color online) Particle energy growth for six different values of the magnetic field, $B=0.0, B=0.20, B=0.31$, $B=0.32, B=0.45$, and $B=0.60$ in terms of the collision number $(n)$. We observe that FA occurs for null and small fields. We used the parameters $\delta=0.30, r_{0}=0.45, e_{R}=e_{r}=0.01, \omega=1.0$, and $\phi_{e}-\phi_{i}=0$.

collisions with the external boundary. All the other parameters are the same as Fig. 4. We can observe that there is some roughness in the curves of the mean velocities but all of them saturate and no FA is observed, which corroborates with Fig. 4.

The explanation for this behavior can be gotten from the radius of curvature of the trajectories due to the magnetic field. The oscillations of the boundaries make this radius no longer

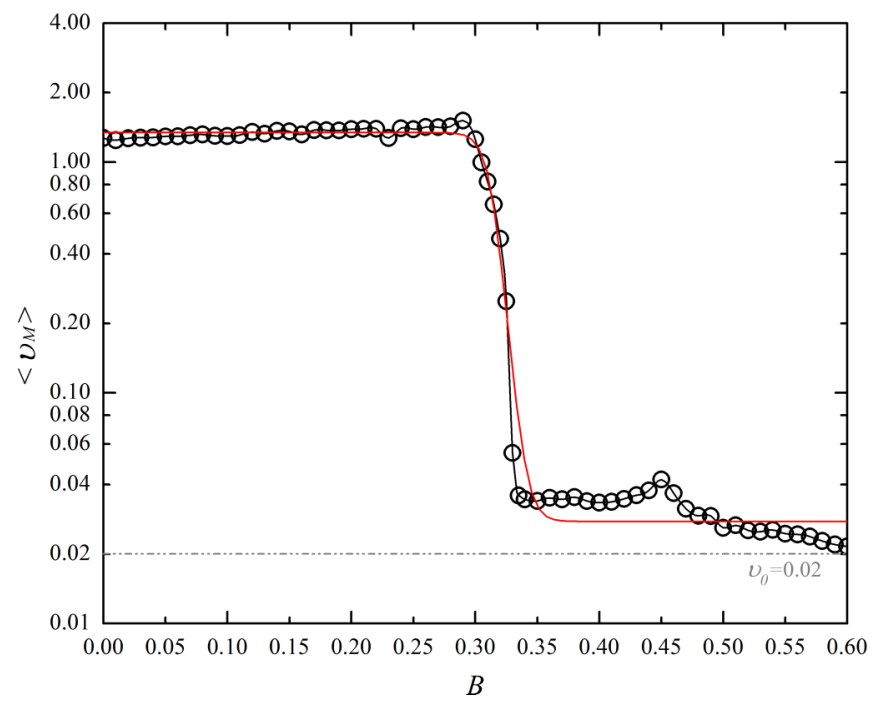

FIG. 5. (Color online) Eccentric MPAB. Mean velocities at the $10^{5}$-th collision with the external boundary for 65 different values of $B$. The dash-dotted line corresponds to the initial particle's velocity and it is fixed as $v_{0}=0.02$. The difference between the initial velocity and the plotted ones shows how much the particle can gain energy depending on the magnitude of the magnetic field. For strong fields the particle keeps its velocity nearby the initial value $v_{0}$. The red curve is a numeric fitting while the gray one is only to guide the eyes.

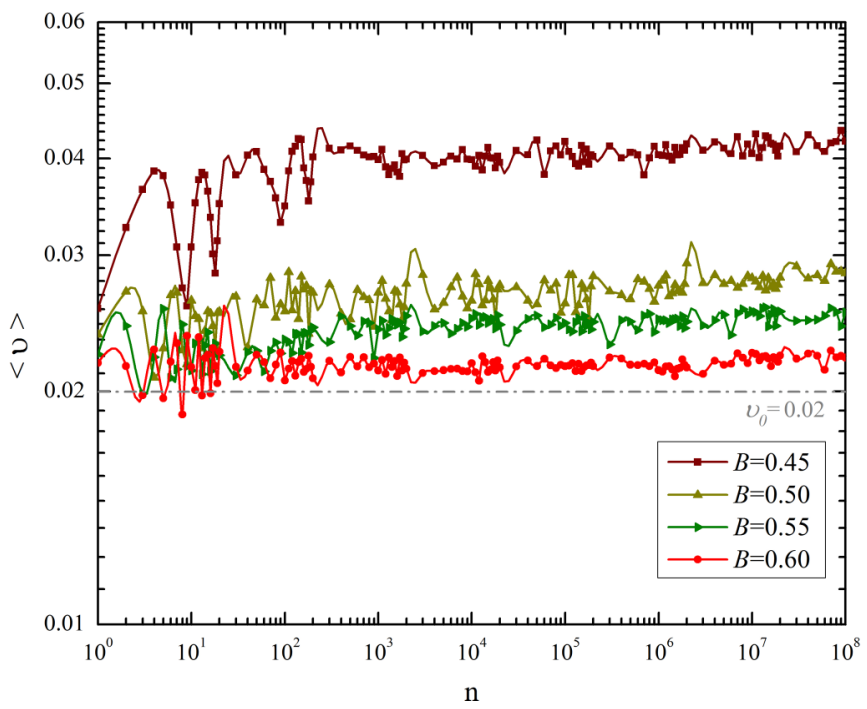

FIG. 6. (Color online) Evolution of the mean velocity up $10^{8}$ iterations for 500 initial conditions. The fields considered are $B=0.45, B=0.50, B=0.55$, and $B=0.60$. We can see the total suppression of FA for all values of the magnetic field.

constant since in each hit with the boundaries the velocity of the particle is altered. For intermediate values of $B$, depending on how the fluctuations of the radius of the particle trajectory occur, one establishes the suppression or the maintenance of the FA. Considering weak magnitudes of $B$, the mechanism of FA is observed. On the other hand, for strong fields the particle tends to stay confined in the region of whispering gallery orbits, which is an annulus between the outer circumference with radius $\left(R_{0} \pm e_{R}\right)$ and the auxiliary circumference of radius $\left(r_{0} \pm e_{r}+\delta\right)$, in such way that it will not collide, or will rarely collide, with the internal boundary even after a huge number of iterations. Therefore, even considering a chaotic static regime and introducing a periodic time-dependent perturbation on the billiard boundaries, the mechanism of FA can be totally deactivated without introducing any kind of dissipation. This also shows that FA can be a tunable mechanism depending on an external perturbation.

\section{ACKNOWLEDGMENT}

We thank the Brazilian scientific agency FAPESP-São Paulo Research Foundation for financial support through Grants No. 2010/20276-2 and No. 2011/14460-8.

\section{APPENDIX: DERIVATION OF THE HAMILTONIAN EQUATION (2) AND ASSOCIATED EQUATIONS}

The kinetic and the generalized velocity-dependent potential energies of a charged particle, with charge $q$ and mass $m$, under the effect of a constant magnetic field, $\vec{B}=B \hat{z}$, are respectively, given by

$$
T=\frac{1}{2} m v^{2}
$$

and

$$
U=-\frac{q}{c} \frac{d \vec{r}}{d t} \cdot \vec{A}(\vec{r}, t)
$$


where $c$ is the light velocity, $\vec{A}(\vec{r}, t)$ is the magnetic vector potential, and $\vec{r}$ is the particle position into the plane $(x, y)$, which is written as

$$
\vec{r}=x \hat{x}+y \hat{y},
$$

and $\vec{v}=\frac{d \vec{r}}{d t}$ is the total velocity of the particle.

The Lagrangian is then written as

$$
L=\frac{1}{2} m v^{2}+\frac{q}{c} \frac{d \vec{r}}{d t} \cdot \vec{A}(\vec{r}, t),
$$

with generalized (or canonical) momenta

$$
p_{k}=m v_{k}+\frac{q}{c} A_{k},
$$

and the corresponding Hamiltonian is

$$
H=\sum_{k} p_{k} v_{k}-L=m v^{2}+\frac{q}{c} \vec{v} \cdot \vec{A}-L,
$$

or, substituting (A4) it is simply

$$
H=\frac{1}{2} m v^{2}
$$

which is the total energy of the particle.

In terms of the generalized momenta, the Hamiltonian is then,

$$
H=\frac{1}{2 m}\left(\vec{p}-\frac{q}{c} \vec{A}\right)^{2}
$$

In polar coordinates, $(\rho, \theta), \vec{r}$, and the total velocity, $\vec{v}=\frac{d \vec{r}}{d t}$, are described as

$$
\vec{r}=\rho \cos \theta \hat{x}+\rho \sin \theta \hat{y}
$$

and

$$
\begin{aligned}
\vec{v}=\frac{d \vec{r}}{d t} & =\dot{\rho}[\cos \theta \hat{x}+\sin \theta \hat{y}]+\rho \dot{\theta}[\cos \theta \hat{y}-\sin \theta \hat{x}] \\
& =\dot{\rho} \hat{\rho}+\rho \dot{\theta} \hat{\theta}=v_{\rho} \hat{\rho}+v_{\theta} \hat{\theta} .
\end{aligned}
$$

From there,

$$
v^{2}=\frac{d \vec{r}}{d t} \cdot \frac{d \vec{r}}{d t}=\dot{\rho}^{2}+\rho^{2} \dot{\theta}^{2},
$$

and the kinetic energy is

$$
T=\frac{m}{2}\left(\dot{\rho}^{2}+\rho^{2} \dot{\theta}^{2}\right)
$$

Considering the asymmetric gauge, $A_{\theta}=\frac{1}{2} B \rho$, the potential vector is given by

$$
\vec{A}=A_{\theta} \hat{\theta}=\frac{B \rho}{2} \hat{\theta} ;
$$

hence,

$$
U=-\frac{q B \rho^{2}}{2 c} \dot{\theta} .
$$

Then the Lagrangian of the system in polar coordinates is

$$
L=\frac{m}{2}\left(\dot{\rho}^{2}+\rho^{2} \dot{\theta}^{2}\right)+\left(\frac{q B \rho^{2}}{2 c} \dot{\theta}\right),
$$

and the generalized momenta are

$$
\begin{gathered}
p_{\theta}=\frac{\partial L}{\partial \dot{\theta}}=m \rho^{2} \dot{\theta}+\frac{q B}{2 c} \rho^{2}, \\
p_{\rho}=\frac{\partial L}{\partial \dot{\rho}}=m \dot{\rho},
\end{gathered}
$$

which are read as follows: $p_{\theta}$ is the angular momentum of the particle and $p_{\rho}$ is the linear momentum along $\rho$. From these equations we get

$$
\begin{gathered}
\dot{\rho}=\frac{p_{\rho}}{m}, \\
\dot{\theta}=\frac{1}{m \rho^{2}}\left(p_{\theta}-\frac{q B}{2 c} \rho^{2}\right) .
\end{gathered}
$$

The Hamiltonian function of the system is obtained from the generalized momenta $\left(p_{k}\right)$ and position $\left(q_{k}\right)$ and the Lagrangian (A15),

$$
H=\sum_{k} p_{k} \dot{\mathrm{q}}_{k}-L,
$$

$$
\begin{gathered}
H=\left(p_{\rho} \dot{\rho}+p_{\theta} \dot{\theta}\right)-\frac{m}{2}\left(\dot{\rho}^{2}+\rho^{2} \dot{\theta}^{2}\right)-\left(\frac{q B \rho^{2}}{2 c} \dot{\theta}\right), \\
H=\dot{\rho}\left(p_{\rho}-\frac{m}{2} \dot{\rho}\right)+\dot{\theta}\left(p_{\theta}-\frac{m}{2} \rho^{2} \dot{\theta}-\frac{q B \rho^{2}}{2 c}\right) .
\end{gathered}
$$

Now, we substitute the expression for $\dot{\rho}$ (A18) and $\dot{\theta}$ (A19) into (A22) and we easily get

$$
H=\frac{1}{2 m}\left[p_{\rho}^{2}+\frac{1}{\rho^{2}}\left(p_{\theta}-\frac{q B}{2 c} \rho^{2}\right)^{2}\right] .
$$

The same result follows when we substitute (A18) and (A19) into (A12). The corresponding Hamilton's equations are

$$
\dot{\rho}=\frac{\partial H}{\partial p_{\rho}}=\frac{p_{\rho}}{m},
$$

$$
\begin{aligned}
\dot{p}_{\rho}=-\frac{\partial H}{\partial \rho}= & -\frac{1}{2 m}\left\{\frac{-2}{\rho^{3}}\left(p_{\theta}-\frac{q B}{2 c} \rho^{2}\right)^{2}\right. \\
& \left.+\frac{1}{\rho^{2}}\left[2\left(p_{\theta}-\frac{q B}{2 c} \rho^{2}\right)\left(-\frac{q B}{c} \rho\right)\right]\right\} \\
= & \frac{1}{m \rho^{3}}\left[\left(p_{\theta}-\frac{q B}{2 c} \rho^{2}\right)\left(p_{\theta}+\frac{q B}{2 c} \rho^{2}\right)\right] \\
= & \frac{1}{m \rho^{3}}\left[p_{\theta}^{2}-\left(\frac{q B}{2 c} \rho^{2}\right)^{2}\right], \\
\dot{\theta}= & \frac{\partial H}{\partial p_{\theta}}=\frac{1}{m \rho^{2}}\left(p_{\theta}-\frac{q B}{2 c} \rho^{2}\right),
\end{aligned}
$$

$$
\dot{p}_{\theta}=-\frac{\partial H}{\partial \theta}=0 \Rightarrow p_{\theta}=\text { constant of motion. }
$$


This constant of motion is a consequence of the chosen gauge. The boundary conditions can also break down this constant. For the eccentric circles, a natural dependence on $\theta$ is introduced. These equations of motion are numerically integrated, with the time-dependent boundary conditions, and we get $\left(\rho, p_{\rho}, \theta, p_{\theta}\right)$ for each iteration of all initial conditions.
The squared total particle velocity, between two successive collisions with the boundaries, is computed through (A11), $v^{2}=\dot{\rho}^{2}+\rho^{2} \dot{\theta}^{2}$, in such way that the right sides of (A24) and (A26) give $\dot{\rho}$ and $\dot{\theta}$, respectively. Immediately after a collision, the radial velocity is properly corrected and the procedure continues.
[1] A. Loskutov, A. B. Ryabov, and L. G. Akinshin, J. Phys. A 33, 7973 (2000).

[2] A. Loskutov and A. B. Ryabov, J. Stat. Phys. 108, 995 (2002).

[3] R. E. de Carvalho, F. C. Souza, and E. D. Leonel, Phys. Rev. E 73, 066229 (2006).

[4] R. Egydio de Carvalho, F. C. Souza, and E. D. Leonel, J. Phys. A 39, 3561 (2006).

[5] E. D. Leonel and P. V. E. McClintock, J. Phys. A 38, 823 (2005).

[6] A. K. Karlis, P. K. Papachristou, F. K. Diakonos, V. Constantoudis, and P. Schmelcher, Phys. Rev. Lett. 97, 194102 (2006); Phys. Rev. E 76, 016214 (2007).

[7] F. Lenz, F. K. Diakonos, and P. Schmelcher, Phys. Rev. Lett. 100, 014103 (2008).

[8] E. D. Leonel and L. A. Bunimovich, Phys. Rev. Lett. 104, 224101 (2010).

[9] V. Gelfreich, V. Rom-Kedar, K. Shah, and D. Turaev, Phys. Rev. Lett. 106, 074101 (2011).

[10] R. S. Pinto and P. S. Letelier, Phys. Lett. A 375, 3273 (2011).

[11] V. Gelfreich, V. Rom-Kedar, and D. Turaev, Chaos 22, 033116 (2012).

[12] A. B. Ryabov and A. Loskutov, Chaos 22, 026121 (2012).
[13] A. Kamor, F. Mauger, C. Chandre, and T. Uzer, Phys. Rev. E 85, 016204 (2012).

[14] M. Hoshino, Phys. Rev. Lett. 108, 135003 (2012).

[15] G. Kowal, E. M. de Gouveia Dal Pino, and A. Lazarian, Phys. Rev. Lett. 108, 241102 (2012).

[16] A. K. Karlis, F. K. Diakonos, C. Petri, and P. Schmelcher, Phys. Rev. Lett. 109, 110601 (2012).

[17] N. Nishizuka and K. Shibata, Phys. Rev. Lett. 110, 051101 (2013).

[18] N. H. Bian and E. P. Kontar, Phys. Rev. Lett. 110, 151101 (2013).

[19] C. Dembowski, H.-D. Gräf, A. Heine, R. Hofferbert, H. Rehfeld, and A. Richter, Phys. Rev. Lett. 84, 867 (2000).

[20] R. Egydio de Carvalho, C. Vieira Abud, and F. Caetano Souza, Phys. Rev. E 77, 036204 (2008).

[21] C. V. Abud and R. Egydio de Carvalho, Phys. Rev. E 84, 036204 (2011).

[22] R. Egydio de Carvalho and C. Vieira Abud, Chaos Solitions Fractals 44, 569 (2011).

[23] C. Vieira Abud and R. Egydio de Carvalho, Phys. Rev. E 88, 042922 (2013).

[24] P. U. Jepsen, B. M. Fischer, A. Thoman, H. Helm, J. Y. Suh, R. Lopez, and R. F. Haglund, Jr., Phys. Rev. B 74, 205103 (2006). 